\title{
Are joints affected by gout also affected by osteoarthritis?
}

\author{
Edward Roddy, Weiya Zhang, Michael Doherty
}

Ann Rheum Dis 2007;66:1374-1377. doi: 10.1136/ard.2006.063768

See end of article for authors' affiliations

......................

Correspondence to:

Edward Roddy, Primary

Care Musculoskeletal

Research Centre, Primary

Care Sciences, Keele

University, Staffordshire, ST5

5BG; e.roddy@cphc.keele.

ac.uk

Accepted 28 January 2007

Published Online First

7 February 2007

.....................
Objectives: To determine whether joints affected by gout are also affected by osteoarthritis (OA).

Methods: A postal questionnaire was sent to all adults aged over 30 years registered with two general practices. The questionnaire assessed a history of gout (doctor diagnosed, or episodes suggestive of acute crystal synovitis) and medication use. Patients who possibly had gout attended for clinical assessment to verify the diagnosis on clinical grounds and assess the distribution of joints affected by acute attacks of gout and OA. Adjusted odds ratios (aOR) and 95\% confidence intervals (Cl) were calculated between the history of an acute attack of gout and the presence of $\mathrm{OA}$ at an individual joint adjusted for age, gender, body mass index and prior diuretic use in a binary logistic regression model.

Results: A total of 4249 completed questionnaires were returned (32\%). From 359 attendees, 164 cases of gout were clinically confirmed. A highly significant association existed between the site of acute attacks of gout and the presence of $\mathrm{OA}(\mathrm{aOR} 7.94 ; 95 \% \mathrm{Cl} 6.27,10.05)$. Analysis at individual joint sites revealed a significant association at the first metatarsophalangeal joint (aOR 2.06; $95 \% \mathrm{Cl} 1.28,3.30$ ), mid-foot (aOR $2.85 ; 95 \% \mathrm{Cl} 1.34,6.03)$, knee (aOR $3.07 ; 95 \% \mathrm{Cl} 1.05,8.96)$ and distal interphalangeal joints (aOR $12.67 ; 95 \% \mathrm{Cl} 1.46,109.911$.

Conclusion: Acute attacks of gout at individual joint sites are associated with the presence of clinically assessed $O A$ at that joint suggesting that $O A$ may predispose to the localised deposition of monosodium urate crystals.

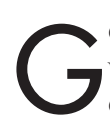
out is one of the most common inflammatory arthritides with a community prevalence of $1-2 \% .^{12}$ It displays a characteristic pattern of joint involvement, in particular, a striking tendency to affect the first metatarsophalangeal (1st MTP) joint. It tends to affect also distal peripheral joints such as the mid-tarsal joints, ankles, knees, interphalangeal (IP) finger joints, wrists and elbows, with axial joints such as the hips, shoulders and spine only very rarely affected. The reason for this characteristic distribution of affected joints is not understood. However, case reports and small hospital-based case series report the occurrence of gout at joint sites affected by osteoarthritis (OA) especially the lst MTP joint and distal IP finger joints; ${ }^{3-7}$ this suggests that changes in the osteoarthritic joint may predispose to monosodium urate (MSU) crystal formation. ${ }^{8}$ Furthermore, a Polish hospital-based study of 262 subjects with gout found an association between gout and radiographic evidence of OA at the lst MTP joints, tarsal joints and knees. ${ }^{9}$ This study did not, however, consider upper limb joints and also runs the risk of inherent bias as a hospital-based population, whereas gout is managed largely in primary care.

We undertook this community-based study in order to determine whether joints affected by gout are also affected by OA, both overall and at individual joint sites.

\section{METHODS}

The study was undertaken in two general practices in Nottingham, nested within a case-control study. It consisted of two phases: (1) a postal questionnaire survey, and (2) a faceto-face clinical assessment. The study was approved by the Nottingham Local Research Ethics Committee 2 and written consent to participate was obtained from participants who attended for clinical assessment.

\section{Postal questionnaire}

Each practice drew up a list of all registered adults over the age of 30 years, excluding those with a history of major psychiatric disease, dementia or recently diagnosed malignancy.
A questionnaire was then mailed to all listed individuals with a pre-paid envelope included for their reply. Possible cases of gout were identified by two questions:

- Have you ever been diagnosed with or suffered from gout?

- Have you ever suffered from an acute attack of arthritis that was severely painful, was associated with a red and swollen joint, came on suddenly reaching its peak severity within $24 \mathrm{~h}$ and then went away completely within 3 weeks? (ie, a typical attack of crystal synovitis)?

Age of onset of gout, current use of allopurinol and diuretic use prior to the onset of gout were requested in addition to recording standard demographic information, weight and height.

Following the first round of mailing, in order to enhance a poor response rate, an abbreviated questionnaire was mailed out to non-respondents in one practice.

\section{Clinical assessment}

All subjects that indicated a previous diagnosis of gout or a history of acute self-limiting attacks of painful, red, swollen joints in their questionnaire (ie, potential cases) were invited to attend a clinical assessment. At this visit, the subject's joint problems were reviewed by a physician with special training in gout (ER) and the most likely diagnosis was made on clinical grounds. The diagnosis of gout was further assessed according to American Rheumatism Association preliminary criteria for the acute arthritis of primary gout. ${ }^{10}$ Each subject was asked where (ie, individual joint sites) they had ever experienced acute attacks of gout, for example, lst MTP joint, mid-foot, ankle, knee, hip, finger and thumb IP joints, thumb-base (ie, first carpo-metacarpal joint), wrist, elbow or shoulder (36 joints

Abbreviations: aOR, adjusted odd ratio; $\mathrm{BMI}$, body mass index $\mathrm{Cl}$, confidence interval; DIP, distal interphalangeal; IP, interphalangeal; MSU, monosodium urate; MTP, metatarsophalangeal; OA, osteoarthritis; OR, odds ratio; PIP, proximal interphalangeal 
in total for each subject). Subjects were also asked to specify whether attacks had affected the left or right side at each individual joint site.

Subjects were systematically examined for the presence of OA at all joint sites. OA of the hip was considered to be present if the subject had had a hip replacement or there was restriction of passive internal rotation of the hip with the hip flexed at $90^{\circ}$. The knee joint was examined for five features: joint replacement, restricted passive flexion and extension, crepitus, joint line tenderness and effusion. OA of the knee was defined according to two criteria: (1) the subject had had a knee replacement, or (2) there was restricted passive flexion/ extension with either crepitus or joint line tenderness. At other joint sites, OA was defined as the presence of restricted passive movement or bony swelling or crepitus.

Participants were also examined for the presence of tophi. These were classified as definite (asymmetric white/yellow swellings on pulling the overlying skin taut) or possible (asymmetric nodular swellings without white/yellow discoloration). Blood was taken for measurement of serum urate and creatinine.

\section{Statistical analysis}

The unit of analysis was considered to be the joint rather than the subject. Two analyses were undertaken.

First, the crude odds ratio (OR) and 95\% confidence interval (CI) between the presence of OA at an individual joint and a history of an acute attack of gout at that joint were calculated for all joint sites combined. This analysis included 5904 individual joints (ie, all 36 joints examined in 164 individuals). Whether a joint had been affected by an acute attack of gout was then entered as the dependent variable into a forward stepwise logistic regression model with age (decades), gender, body mass index (BMI; $\left.>30 \mathrm{~kg} / \mathrm{m}^{2}\right)$, previous diuretic use and presence of OA at that joint as independent variables and an adjusted OR (aOR) and 95\% CI were calculated. In order to investigate the influence of disease duration on the relationship between the site of acute attacks of gout and the presence of OA, subjects were grouped according to tertiles of disease duration and the crude and adjusted ORs calculated for each tertile.

Secondly, crude and adjusted ORs were then calculated at each individual joint site (left and right sides combined). As acute attacks of gout in the finger IP joints were an uncommon occurrence, analyses were performed for three anatomical units: distal IP (DIP) joint row, proximal IP (PIP) joint row and the whole hand (ie, acute attacks of gout and OA occurring anywhere within that unit).

\section{RESULTS}

Questionnaires were mailed to a total of 13684 individuals, and 4249 completed responses were returned; 294 were notified as being sent to the wrong address. The adjusted response rate was, therefore, $32 \%$ (fig 1 ).

A total of 488 respondents reported acute attacks of selflimiting synovitis and/or a diagnosis of gout: 264 had been given a diagnosis of gout. Three hundred and fifty-nine attended the clinical assessment (75\%). Of these, 175 reported being diagnosed with gout by a doctor. This was thought to be the correct diagnosis in 145 (83\%). A further 19 subjects were thought to have gout but had never consulted a doctor. Therefore, 164 subjects were identified as having gout. Characteristics of the 164 subjects with gout are shown in table 1 .

The most common site of acute attacks of gout was the lst MTP joint followed by the mid-foot, ankle and knee (table 2).
Upper limb involvement was uncommon. Involvement of left and right sides was similar at all joint sites.

Analysis of all 5904 individual joint sites combined found a highly significant association between the site of acute attacks of gout and the presence of OA after adjustment for confounding factors (aOR 7.94; 95\% CI 6.27, 10.05) (table 3).

On indirect comparison of the three tertiles of disease duration, the strength of association between sites of acute attacks of gout and the presence of OA appeared similar (table 4). ORs were numerically smaller with increasing disease duration, although there was considerable overlap of 95\% CI.

Analysis at individual joint sites revealed a significant association between acute attacks of gout and the presence of OA at the 1st MTP joint (aOR 2.06; 95\% CI 1.28, 3.30), mid-foot (aOR 2.85; 95\% CI 1.34, 6.03), knee (aOR 3.07; 95\% CI 1.05, 8.96) and DIP joints (aOR 12.67; 95\% CI 1.46, 109.91) after adjustment for confounding variables (table 5 ).

\section{DISCUSSION}

A strong association was seen between joint sites affected by acute attacks of gout and the presence of clinically assessed OA. When this relationship was assessed at individual joint sites, significant associations were seen at the 1st MTP, mid-foot, knee and finger DIP joints after adjustment for confounding variables. These data support the hypothesis that the presence of OA at an individual joint site predisposes to the formation of urate crystals at that site as suggested by a number of case reports and small case series ${ }^{3-7}$ and one Polish hospital-based study. ${ }^{9}$ This latter study found a significant correlation between gout and radiographic presence of OA at the 1st MTP joint, midfoot and knee in 262 subjects with gout. The current study is the first to evaluate this question in a community-based sample and to consider the upper limb joints.

A number of mechanisms have been suggested to explain an association between the sites of acute attacks of gout and OA, including: mechanical shock; ${ }^{11}$ changes in cartilage and synovial proteoglycans; ${ }^{12}$ epitaxial MSU crystal formation on cartilage fragments; ${ }^{13}$ and transient increases in the urate concentration of resolving synovial effusions owing to the differential permeability of synovium to urate and water, ${ }^{8}$ which might encourage nucleation and precipitation of MSU crystals. An association between $\mathrm{OA}$ and calcium crystal deposition is well recognised. Community studies have confirmed a positive association between knee OA and chondrocalcinosis. ${ }^{14}$ There is an increased incidence of calcium pyrophosphate dihydrate and basic calcium phosphate crystals in synovial fluid and cartilage from OA joints, ${ }^{15}$ and also local mechanical factors (including joint hypermobility ${ }^{16}$ and instability, ${ }^{17}$ joint motion, ${ }^{18}$ ligamentous laxity ${ }^{19}$ and joint damage from surgery ${ }^{20}{ }^{21}$ and trauma ${ }^{22}$ ) are known to predispose to localised secondary deposition of calcium pyrophosphate dihydrate crystals in the context of joint damage. It is possible that OA results in changes in tissue factors, either an increase in promoters or a reduction in inhibitors of crystal nucleation and growth, which might predispose not just to calcium crystal but also to MSU crystal deposition. It has also been suggested that the predilection of gouty arthritis and tophi for cooler extremities such as the hallux, fingers and ears and the rarity of gout at axial joints such as the hip and shoulder could be explained by the reduction seen in the solubility of urate with decreasing temperature. ${ }^{23}{ }^{24}$ However, this does not explain why gout should preferentially affect the MTP joint of the great toe rather than the lesser toes or the great toe IP joint.

The major limitation to our study is the low overall response rate to the postal questionnaire. In addition to 164 gout cases identified, there were a further 89 subjects who reported suffering from gout in the questionnaire but did not consent to 


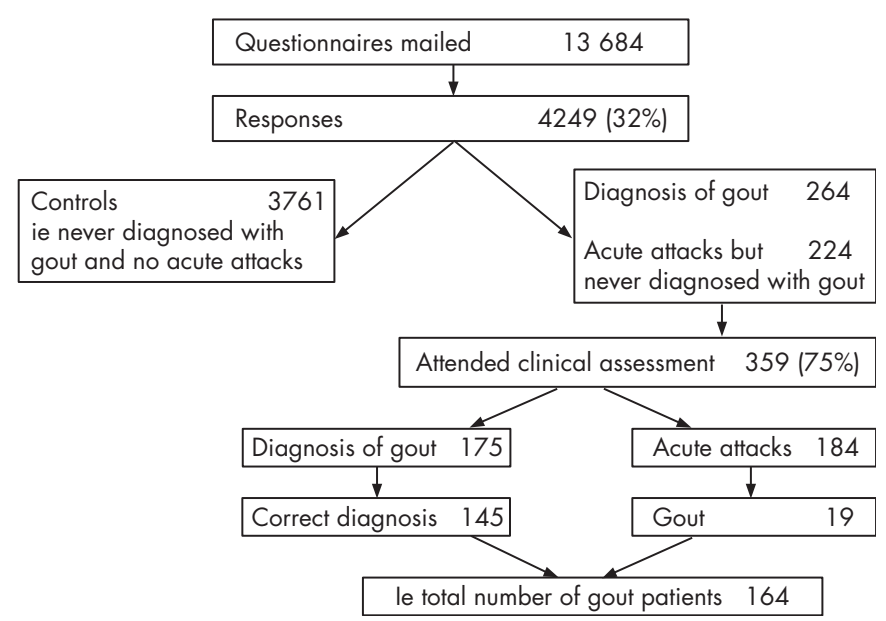

Figure 1 Flow of subjects through the study.

clinical assessment. Another possible limitation is the use of a clinical case definition for gout. The recent EULAR (the European League Against Rheumatism) recommendations for the diagnosis of gout found that the rapid development of severe pain, swelling, tenderness and erythema is highly suggestive of crystal inflammation, although not specific for gout; ${ }^{25}$ however, 1st MTP joint involvement markedly increases the specificity of this clinical composite. Although it is possible that some of the acute attacks that were presumed to be due to gout were, in fact, due to other crystals, most commonly calcium pyrophosphate; this is unlikely to have accounted for much misclassification given the sites of involvement observed. Without doubt, the gold standard for the diagnosis of gout is the identification of crystals on aspirates from synovial fluid or tophus. However, while intercritical joint aspiration is a useful diagnostic technique, ${ }^{26}$ the requirement to undergo aspiration may have reduced further the number of individuals

Table 1 Characteristics of subjects with gout

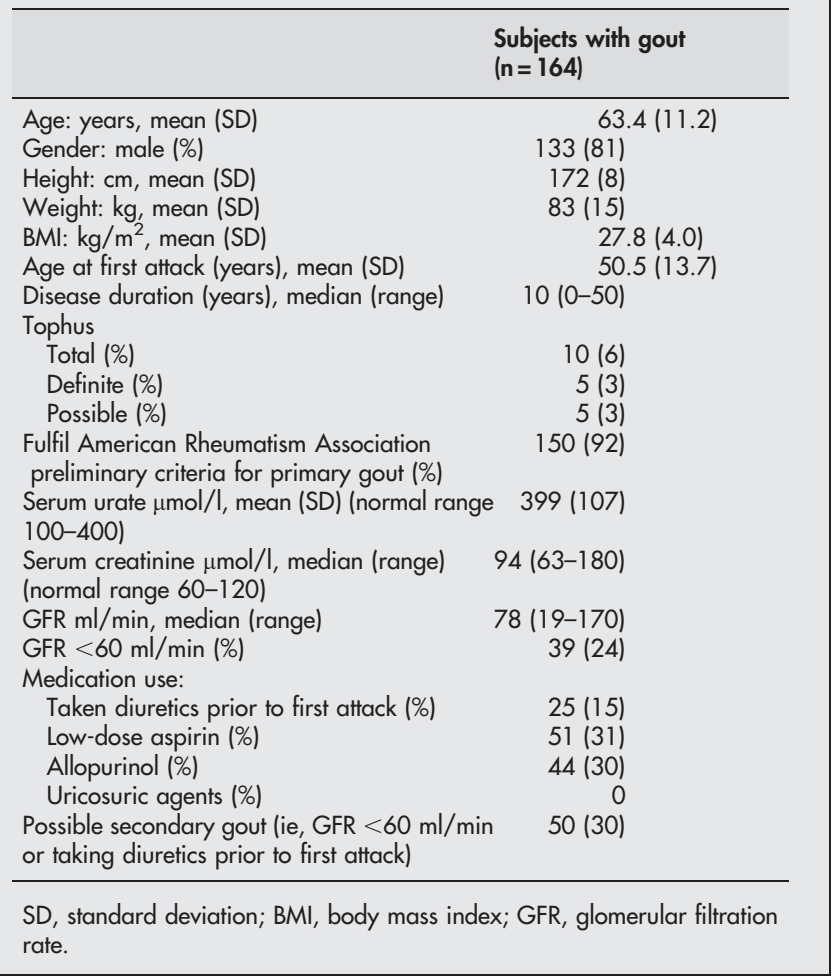

Table 2 frequency of acute attacks of gout at each joint site

\begin{tabular}{lll}
\hline Joint & Left (\% of subjects) & Right (\% of subjects) \\
\hline 1st MTP joint & 61 & 66 \\
Mid-foot & 13 & 20 \\
Ankle & 12 & 15 \\
Knee & 7 & 12 \\
Hip & 0 & 0 \\
DIP joints & 2 & 2 \\
PIP joints & 1 & 1 \\
Thumb-base & 1 & 1 \\
Hand & 4 & 3 \\
Wrist & 3 & 4 \\
Elbow & 3 & 4 \\
Shoulder & 1 & 1 \\
\hline
\end{tabular}

MTP, metatarsophalangeal; DIP, distal interphalangeal; PIP, proximal interphalangeal.

Table 3 Odds ratio (OR) and 95\% confidence interval (Cl) between the site of acute attacks of gout and presence of $O A$

\begin{tabular}{llll}
\hline Variable & Crude OR $(95 \% \mathrm{Cl})$ & $\begin{array}{l}\text { Adjusted OR } \\
(95 \% \mathrm{Cl})^{*}\end{array}$ & $\mathbf{P}$ \\
\hline $\begin{array}{l}\text { Presence of } \\
\text { OA in the }\end{array}$ & $6.76(5.39,8.46)$ & $7.94(6.27,10.05)$ & $<0.001$ \\
index joint & & & \\
\hline
\end{tabular}

*Adjusted for age (decades), gender, BMI $\left(>30 \mathrm{~kg} / \mathrm{m}^{2}\right)$ and prior diuretic use using a forward step-wise binary logistic regression model with gout in the index joint as the dependent variable.

Table 4 Odds ratios (OR) and $95 \%$ confidence interval (Cl) between attacks of gout and presence of OA stratified by disease duration (tertiles)

\begin{tabular}{lcll}
\hline & $\begin{array}{l}\text { Disease duration } \\
\text { (years), mean (SD) }\end{array}$ & Crude OR (95\% Cl) & $\begin{array}{l}\text { Adjusted OR } \\
(95 \% \mathrm{CI})^{*}\end{array}$ \\
\hline Tertile 1 & $2.9(2.2)$ & $7.39(4.80,11.37)$ & $10.12(6.29,16.29)$ \\
Tertile 2 & $10.2(2.1)$ & $7.19(4.97,10.41)$ & $8.22(5.61,12.06)$ \\
Tertile 3 & $25.9(8.4)$ & $6.02(4.11,8.80)$ & $6.45(4.38,9.48)$ \\
\hline
\end{tabular}

*Adjusted for age (decades), gender, BMI $\left(>30 \mathrm{~kg} / \mathrm{m}^{2}\right)$ and prior diuretic use using a forward step-wise binary logistic regression model with gout in the index joint as the dependent variable.

Table 5 Odds ratio (OR) and $95 \%$ confidence interval (Cl) between acute attacks of gout and presence of OA for each joint site (left and right combined)

\begin{tabular}{lccl}
\hline Joint & Crude OR $(95 \% \mathrm{Cl})$ & Adjusted OR $(95 \% \mathrm{CI})^{*} \mathbf{p}$ \\
\hline 1st MTP joint & $1.73(1.10,2.73)$ & $2.06(1.28,3.30)$ & 0.003 \\
Mid-foot & $2.58(1.24,5.35)$ & $2.85(1.34,6.03)$ & 0.006 \\
Ankle & $4.08(0.94,17.73)$ & NS & - \\
Knee & $2.98(1.02,8.68)$ & $3.07(1.05,8.96)$ & 0.040 \\
Hip & - & - & - \\
DIP joints & $12.50(1.44,108.46)$ & $12.67(1.46,109.91)$ & 0.021 \\
PIP joints & - & - & - \\
Thumb-base & - & - & - \\
Hand & $2.90(0.86,9.83)$ & NS & - \\
Wrist & $1.16(0.14,9.37)$ & NS & - \\
Elbow & - & - & - \\
Shoulder & - & - & - \\
\hline
\end{tabular}

*Adjusted for age (decades), gender, BMI $\left(>30 \mathrm{~kg} / \mathrm{m}^{2}\right)$ and prior diuretics using a forward step-wise binary logistic regression model with gout in the index joint as the dependent variable.

NS, not significant; MTP, metatarsophalangeal; DIP, distal interphalangeal; PIP, proximal interphalangeal. 
consenting to the clinical assessment, particularly as only $4 \%$ had experienced joint aspiration previously (data not shown). Many studies of gout have used the 1977 American Rheumatism Association preliminary criteria as diagnostic criteria. ${ }^{10}$ However, these classification criteria are limited to the acute arthritis of primary gout, for example, they are not helpful for either chronic gout or secondary gout, and have never undergone further validation as suggested by the authors in the original report. We have, however, included them as a secondary diagnostic criterion. A further similar caveat is that the presence of OA at an individual joint site was diagnosed on the basis of clinical examination findings. These are not specific for OA and may have introduced misclassification bias. Clinical diagnosis of OA may be valid in certain joints, for example, the knee, lst MTP joint, DIP joints and hip, but may be less appropriate at other sites, for example, the mid-foot, shoulder, wrist or ankle. Undertaking radiographs of all sites of interest would have been impractical; furthermore, standard radiographs are relatively insensitive, especially at certain joint sites. Radiographic evidence of an association between gout and OA at the lst MTP joint, mid-foot and knee has been reported by Kawenoki-Minc et al, ${ }^{9}$ although this hospital-based study did not consider upper limbs. Although clinically detectable bony swelling is characteristic of OA and correlates with radiographic $\mathrm{OA},{ }^{27-29}$ crepitus and reduced range of movement may also occur as a result of joint damage secondary to gout. It could also be argued that this explains the observed association between the sites of acute attacks of gout and the clinical examination findings of OA. However, the strength of this association was not influenced by the chronicity of gout suggesting that joint damage secondary to gout does not explain the observed association, although a prospective study is required to confirm this. A final caveat is that the observer was not blinded to which joints had been affected by gout at the time of the clinical examination. Further work using blind radiographic assessment to determine the presence of OA is warranted.

In summary, the deposition of MSU crystals at individual joint sites is associated with the presence of OA at that joint, particularly at the 1st MTP, mid-foot, knee and finger DIP joints suggesting that OA may predispose to the localised deposition of MSU crystals and in part determine which joints are affected by acute attacks of gout.

\section{ACKNOWLEDGEMENTS}

We would like to thank the staff and patients of Arnold Health Centre and The Calverton Practice in Nottingham, UK.

\section{Authors' affiliations \\ Edward Roddy, Weiya Zhang, Michael Doherty, Academic \\ Rheumatology, University of Nottingham, UK}

Funding: We are grateful for funding from the Arthritis Research Campaign, UK (ICAC grant 14851) and unrestricted financial support from Astra-Zeneca-UK, Glaxo-Smith-Kline-USA and Ipsen, France

Competing interests: None.

\section{REFERENCES}

1 Mikuls TR, Farrar JT, Bilker WB, Fernandes S, Schumacher HR Jr, Saag KG. Gout epidemiology: results from the UK General Practice Research Database, 19901999. Ann Rheum Dis 2005;64:267-72.

2 Harris CM, Lloyd DC, Lewis J. The prevalence and prophylaxis of gout in England. J Clin Epidemiol 1995;48:1153-8.

3 Simkin PA, Campbell PM, Larson EB. Gout in Heberden's nodes. Arthritis Rheum 1983;26:94-7.

4 O'Dell JR. Gout in Heberden's nodes. Arthritis Rheum 1983;26:1413-14.

5 Foldes K, Petersilge CA, Weisman MH, Resnick D. Nodal osteoarthritis and gout: a report of four new cases. Skeletal Radiol 1996;25:421-4.

6 Fam AG, Stein J, Rubenstein J. Gouty arthritis in nodal osteoarthritis. J Rheumatol 1996;23:684-9.

7 Lally EV, Zimmermann B, Ho G Jr, Kaplan SR. Urate-mediated inflammation in nodal osteoarthritis: clinical and roentgenographic correlations. Arthritis Rheum 1989;32:86-90.

8 Simkin PA. The pathogenesis of podagra. Ann Intern Med 1977;86:230-3.

9 Kawenoki-Minc E, Eyman E, Leo W, Werynska-Przybylska J. [Osteoarthrosis and spondylosis in gouty patients. Analysis of 262 cases of gout]. Reumatologia 1974;12:267-7 (In Polish with English abstract).

10 Wallace SL, Robinson H, Masi AT, Decker JL, McCarty DJ, Yu TF. Preliminary criteria for the classification of the acute arthritis of primary gout. Arthritis Rheum 1977;20:895-900.

11 Wilcox WR, Khalaf AA. Nucleation of monosodium urate crystals. Ann Rheum Dis 1975;34:332-9.

12 Perricone E, Brandt KD. Enhancement of urate solubility by connective tissue. I. Effect of proteoglycan aggregates and buffer cation. Arthritis Rheum 1978;21:453-60.

13 Pascual E, Ordonez S. Orderly arrayed deposit of urate crystals in gout suggest epitaxial formation. Ann Rheum Dis 1998;57:255-64.

14 Neame RL, Carr AJ, Muir K, Doherty M. UK community prevalence of knee chondrocalcinosis: evidence that correlation with osteoarthritis is through a shared association with osteophyte. Ann Rheum Dis 2003;62:513-18.

15 Hamilton E, Pattrick M, Hornby J, Derrick G, Doherty M. Synovial fluid calcium pyrophosphate dihydrate crystals and alizarin red positivity: analysis of 3000 samples. Br J Rheumatol 1990;29:101-4.

16 Bird HA, Tribe CR, Bacon PA. Joint hypermobility leading to osteoarthrosis and chondrocalcinosis. Ann Rheum Dis 1978;37:203-11.

17 Settas L, Doherty M, Dieppe P. Localised chondrocalcinosis in unstable joints. $\mathrm{Br}$ Med J (Clin Res Ed) 1982;285:175-6.

18 Fam AG, Schumacher HR Jr, Clayburne G, Villanueva T, Baker D, Jimenez SA Effect of joint motion on experimental calcium pyrophosphate dihydrate crystal induced arthritis. J Rheumatol 1990;17:644-55.

19 Scott D, Bird H, Wright V. Joint laxity leading to osteoarthrosis. Rheumatol Rehabil 1979;18:167-9.

20 Doherty M, Watt I, Dieppe PA. Localised chondrocalcinosis in post-meniscectomy knees. Lancet 1982;i:1207-10.

21 Ellman MH, Vazques LT, Brown NL, Mandel N. Calcium pyrophosphate dihydrate deposition in lumbar disc fibrocartilage. J Rheumatol 1981;8:955-8.

22 de Lange EE, Keats TE. Localized chondrocalcinosis in traumatized joints. Skeletal Radiol 1985; 14:249-56.

23 Kippen I, Klinenberg JR, Weinberger A, Wilcox WR. Factors affecting urate solubility in vitro. Ann Rheum Dis 1974;33:313-17.

24 Loeb JN. The influence of temperature on the solubility of monosodium urate. Arthritis Rheum 1972;15:189-92.

25 Zhang W, Doherty M, Pascual E, Bardin T, Barskova V, Conaghan P, et al. EULAR evidence based recommendations for gout-Part I Diagnosis: Report of a task force of the Standing Committee for International Clinical Studies Including Therapeutics (ESCISIT). Ann Rheum Dis 2006;65:1301-11.

26 Pascual E, Batlle-Gualda E, Martinez A, Rosas J, Vela P. Synovial fluid analysis for diagnosis of intercritical gout. Ann Intern Med 1999;131:756-9.

27 Claessens AAMC, Schouten JSAG, Van Ouwelan FA, Valkenburg HA. Do clinical findings associate with radiographic osteoarthritis of the knee? Ann Rheum Dis 1990;49:771-4.

28 Stern AG, Moxley G, Sudha Rao TP, Disler D, McDowell C, Park M. Utility of digital photographs of the hand for assessing the presence of hand osteoarthritis. Osteoarthritis Cartilage 2004;12:360-5.

29 Thaper A, Zhang W, Wright G, Doherty M. Relationship between Heberden's nodes and underlying radiographic change of osteoarthritis. Ann Rheum Dis 2005;64:1214-16. 